\title{
Observación de la fracción de agua líquida en pilas de combustible tipo PEM de cátodo abierto
}

\author{
Julio Luna (julio.luna@chalmers.se) \\ Chalmers University of Technology, Hörsalsvägen 11, SE-41296 Göteborg, Sweden \\ Ramon Costa Castelló (ramon.costa@upc.edu) \\ Institut de Robòtica i Informàtica Industrial, CSIC-UPC. Llorens i Artigas 4-6, 08028 Barcelona, Spain
}

\section{Resumen}

En este trabajo se diseña un observador no-lineal basado en modos deslizantes para la estimación de la fracción de agua líquida en una pila de combustible tipo PEM de cátodo abierto. La pila de combustible se modela con dos estados dinámicos: la temperatura del stack y la saturación de agua líquida. La estrategia de observación se valida en simulación mediante un ciclo de conducción ARTEMIS.

Palabras clave: pila de combustible, observación, modos deslizantes, modelado.

\section{INTRODUCCIÓN}

A medida que el consumo energético incrementa, la sociedad, la industria y los gobiernos están tomando conciencia de la necesidad de implantar nuevos sistemas de energía sostenibles que puedan reducir los problemas asociados con el uso de los combustibles fósiles. La utilización de dichas fuentes no renovables genera emisiones de carbono y no es sostenible a largo plazo. Lograr este cambio tecnológico y social requiere de importantes inversiones y esfuerzos de investigación enfocados a desarrollar alternativas limpias y sostenibles.

Estudios científicos [5] han concluido que los sistemas de generación de energía basados en hidrógeno son una prometedora solución a la hora de satisfacer las demandas de energía presentes y futuras, sin emisiones de carbono adicionales. El hidrógeno como vector energético posee considerables ventajas comparado con otras soluciones renovables. Además, la utilización de hidrógeno puede ayudar a reducir las emisiones de carbono a la atmósfera y diversificar el mercado de generación energética [12]. Esto es debido principalmente a que el hidrógeno puede ser producido a partir de fuentes primarias de energía renovable (solar y eólica). Adicionalmente, el hidrógeno puede quemarse directamente en turbinas para generar electricidad, obteniendo únicamente agua y calor como subproductos de la combustión o también puede utilizarse para alimentar pilas de combustible y producir energía a partir de reacciones electroquímicas. El hidrógeno también puede ser almacenado a largo plazo, convirtiéndolo en un combustible ideal para transporte de larga distancia y aplicaciones móviles.

Las pilas de membrana de intercambio protónico (PEM), las cuales utilizan hidrógeno como combustible, poseen altas densidades de potencia y operan a temperaturas relativamente bajas. Las pilas de tipo PEM son una de las tecnologías de hidrógeno más prometedoras para aplicaciones automotrices, estacionarias y de ciclo combinado. Investigadores de todo el mundo están dedicando importantes esfuerzos para incrementar la eficiencia, reducir la degradación y disminuir los costes de producción de las pilas de combustible de tipo PEM. Para lograr los objetivos anteriores, las pilas de combustible requieren soluciones de control que tengan en cuenta las complejas dinámicas internas del sistema, las cuales incluyen fenómenos multi-físicos tales como mecánica de fluidos, termodinámica y cinética química.

La eficiencia y la degradación de las pilas de combustible están directamente relacionadas con las condiciones de operación internas. En el campo del control automático, nuevas técnicas de observación [8], diagnosis [11] y control [1] están siendo desarrolladas para mejorar las condiciones de operación de los sistemas basados en pilas PEM. La temperatura del sistema, concentraciones de especies gaseosas, densidad de corriente y contenido de agua son, entre otras, variables que afectan el rendimiento del sistema. Por otra parte, la vida útil de las pilas de combustible se ve drásticamente reducida cuando el sistema se ve sujeto a variaciones de carga inesperadas que pueden provocar situaciones de desabastecimiento de reactantes en las capas catalizadoras.

El modelado juega un importante rol en las áreas de control y monitoreo del estado de salud de las pilas de combustible de tipo PEM. De hecho, a la hora de desarrollar estrategias de control y observación cuyo objetivo sea la mejora de la eficiencia y la durabilidad de los sistemas basados en pilas de combustible, los modelos dinámicos son necesarios. Por otra parte, mediante la utilización de 
modelos matemáticos, es posible reducir los costes asociados con las pruebas de laboratorio.

Cuantificar la degradación en pilas de combustible es complejo. Una de las propuestas de la literatura [18] es el modelado del área efectiva de la reacción electro-química. Esta área efectiva viene determinada por el número de partículas de platino $(\mathrm{Pt})$ activas en el soporte de carbono de la capa catalizadora del cátodo. El número de partículas de $\mathrm{Pt}$ activas depende entre otros de las especificaciones de fabricación del catalizador y del estado de hidratación del cátodo. La única variable disponible para diseñar estrategias de control activas es el estado de hidratación del cátodo, que puede ser obtenido a partir del valor de la fracción de agua líquida [14].

La estimación de los estados internos en pilas de combustible de tipo PEM está directamente relacionada con la implementación de estrategias avanzadas de control para mejorar la eficiencia y durabilidad [7]. Mientras que ciertas medidas son realizables mediante sensores, existen partes del sistema que no son accesibles debido a la estructura de la pila, como por ejemplo la fracción de agua líquida. El uso de modelos no-lineales combinados con estrategias de estimación de estado basadas en modelos es una solución novel a la hora de obtener información del estado interno de la pila de combustible [8]. Adicionalmente, el uso de herramientas matemáticas de observación de estados facilita la reducción del número de sensores y por lo tanto, la reducción su coste asociado [4].

La principal contribución del presente trabajo se basa en el desarrollo de un observador no-lineal basado en modos deslizantes para la estimación de la fracción de agua líquida presente en la pila de combustible de tipo PEM. Para lograr este objetivo, un modelo dinámico de una pila de combustible tipo PEM de cátodo abierto [14] se utiliza para el diseño del observador.

Éste artículo está organizado de la siguiente manera. En la Sección 2 se presentan la descripción del sistema. El modelo de simulación se describe en la Sección 3. La estructura del observador de estados no-lineal se describe en la Sección 4. En la Sección 5 se presenta un caso de simulación y se analizan los resultados obtenidos. Finalmente, la Sección 6 resume las conclusiones de este artículo y se proponen las líneas futuras para continuar con la investigación.

\section{DESCRIPCIÓN DEL SISTEMA}

En este estudio se analizará la pila de combustible (stack) comercial H100 desarrollada por Ho- rizon Fuel Cells Technologies, esta está compuesta por 24 celdas y presenta una potencia nominal de 100 W. Esta pila, mostrada en la Figura 1, es de cátodo abierto y presenta una área activa de $22,5 \mathrm{~cm}^{2}$, es auto-humidifica y refrigerada por aire. Incluye un único ventilador de refrigeración directamente unido a la carcasa de la pila de combustible, que refrigera la pila por convección forzada y, al mismo tiempo proporciona oxígeno al cátodo. Por lo tanto, el suministro de aire del cátodo y la pila de refrigeración no se pueden desacoplar.

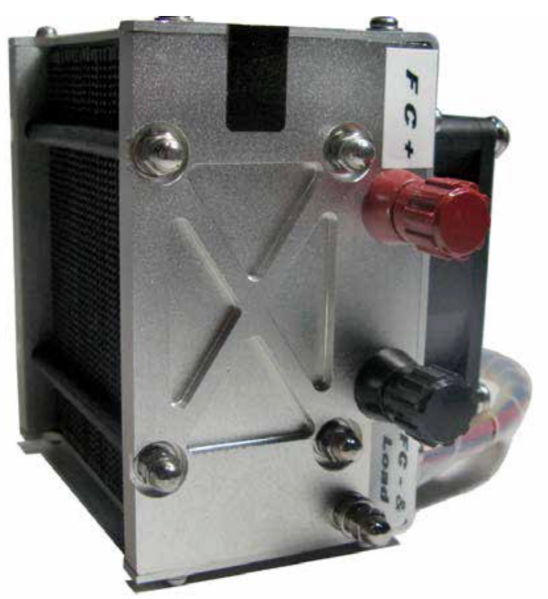

Figura 1: Vista de la H100 [3]

La Figura 2 muestra un esquema del sistema de pila de combustible instalado en una estación de prueba de laboratorio. El ventilador es alimentado mediante fuente de alimentación de $12 \mathrm{~V}$ externa. Un controlador fija la velocidad de giro del ventilador. La velocidad del aire de entrada de cátodo se mediante un sensor de flujo de aire de alta precisión E75 de $E+E$ Elektronik. La entrada del ánodo se alimenta con hidrógeno seco. Un regulador de presión manual mantiene la presión de entrada de ánodo a aproximadamente 0,4 bar. Los parámetros y principales constantes físicas del sistema se incluyen en la Tabla 1.

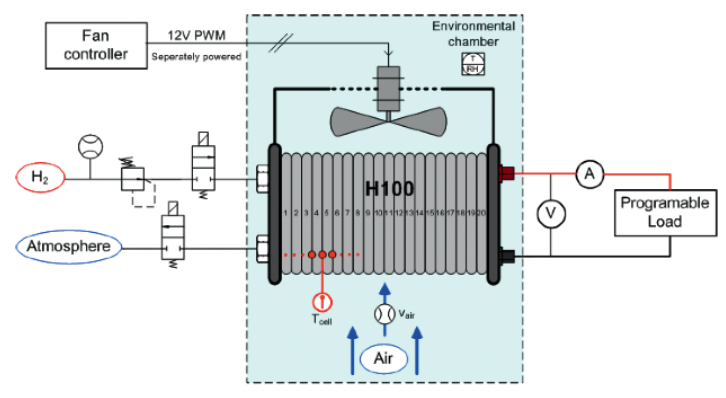

Figura 2: Esquema del sistema para una pila de combustible tipo H100 


\section{MODELO DEL SISTEMA}

El modelo para el sistema descrito en la Sección 2 está descrito por:

$$
\begin{aligned}
\dot{x}_{1} & =K_{1} z_{1} y+K_{1} z_{1}^{2}+K_{2}\left(z_{2}-x_{1}\right) u, \\
\dot{x}_{2} & =K_{3} z_{1}-K_{4} f_{p}\left(x_{1}\right) x_{2}-f_{d}\left(x_{2}\right), \\
y & =K_{5} x_{1} f_{a}\left(x_{1}, x_{2}, z_{1}\right) .
\end{aligned}
$$

Como se puede observar se trata de un modelo de segundo orden. Los estados corresponden a la temperatura de la pila de combustible $\left(x_{1}=T_{f c}\right)$ y la saturación de agua líquida $\left(x_{2}=s\right)$. La saturación corresponde a la relación del volumen de líquido respecto al volumen total de espacio vacío en la estructura porosa. Por lo tanto la saturación describe el contenido de agua líquida en la capa catalizadora [10]. El sistema presenta 3 entradas, la corriente de carga $\left(z_{1}=I_{\text {stack }}\right)$, la temperatura ambiental $\left(z_{2}=T_{a m b}\right)$ y la velocidad de aire $\left(u=v_{\text {air }}\right)$ del ventilador. Esta última suele usarse como acción de control. La salida del sistema, $y=V_{\text {stack }}$, corresponde a la tensión del stack. Así la potencia entregada por el sistema corresponde a $P=y \cdot z_{1}$.

Las Ecuaciones (1)-(2)-(3) presentan forma afín, y son altamente no lineales pues $f_{p}, f_{d}$ y $f_{a}$ lo son. Además la acción de control está multiplicada por funciones que dependen de los estados. El apéndice A contiene el detalle de todas ecuaciones y el valor de todos parámetros.

En trabajos previos $[16,17,15]$ se han analizado en detalle las características dinámicas y estacionarias de este modelo.

\section{OBSERVADOR}

Siguiendo la estructura de observador no lineal propuesta por [2], el observador para el sistema descrito por la Ecuaciones (1)-(2)-(3) se expresa como:

$$
\begin{aligned}
\dot{\hat{x}}_{1} & =K_{1} I-K_{1}^{\prime} I V_{\text {stack }}+\nu+g_{1}(\hat{\mathbf{x}}) u_{1}, \\
\dot{\hat{x}}_{2} & =K_{3} I-K_{4} f_{p}\left(\hat{x}_{1}\right) \hat{x}_{2}-f_{d}\left(\hat{x}_{2}\right)+g_{2}(\hat{\mathbf{x}}) u_{2}, \\
\hat{y} & =h(\hat{\mathbf{x}})=\hat{x}_{1},
\end{aligned}
$$

donde $\hat{\mathbf{x}} \triangleq\left[\hat{x}_{1}, \hat{x}_{2}\right], \hat{x}_{1} \triangleq \hat{T}_{f c}$ y $\hat{x}_{2} \triangleq \hat{s}$. La variable observada de salida es la temperatura de la pila de combustible, descrita por la Ecuación (4c). Donde las funciones $g_{1}(\hat{\mathbf{x}})$ y $g_{2}(\hat{\mathbf{x}})$ y las señales $u_{1}$ y $u_{2}$ son elementos incorporados en el observador para forzar la convergencia de los estados des serán diseñadas para reducir el error de estimación a cero en tiempo finito.

\subsection{Análisis de observabilidad}

En sistemas no lineales, el estudio de la observabilidad depende del rango de la matriz de observabi- lidad $\mathcal{O}[2]$. Para un sistema de orden $n$, la matriz de observabilidad se define como:

$$
\mathcal{O}(\mathbf{x})=\frac{\partial}{\partial \mathbf{x}}\left[\begin{array}{c}
h(\mathbf{x}) \\
L_{f(\mathbf{x})} h(\mathbf{x}) \\
\vdots \\
L_{f(\mathbf{x})}^{n-1} h(\mathbf{x})
\end{array}\right],
$$

donde $n$ es el número de estados en $\mathbf{x}$ y $L_{f(\mathbf{x})} h(\mathbf{x})$ es la derivada de Lie del vector de salida $h$ a lo largo del campo vectorial $f(\mathbf{x}) \in \mathbb{R}^{n}$, definida como [13]:

$$
L_{f(\mathbf{x})} h(\mathbf{x})=\frac{\partial h(\mathbf{x})}{\partial \mathbf{x}} f(\mathbf{x}) .
$$

Y la $k$-ésima derivada de Lie:

$$
L_{f(\mathbf{x})}^{k} h(\mathbf{x})=\frac{\partial\left(L_{f(\mathbf{x})}^{k-1} h(\mathbf{x})\right)}{\partial \mathbf{x}} f(\mathbf{x}) .
$$

Utilizando las Ecuaciones (6) y (7), la matriz de observabilidad para la Ecuación (4) es calculada:

$$
\mathcal{O}(\hat{\mathbf{x}})=\left[\begin{array}{cc}
1 & 0 \\
\xi(\hat{\mathbf{x}}) & \gamma(\hat{\mathbf{x}})
\end{array}\right],
$$

siendo $\xi$ y $\gamma$ funciones no lineales que dependen de los estados del sistema.

Tal y como demuestra [2], para garantizar la observabilidad de la Ecuación (4), el rango de $\mathcal{O}$ debe ser igual a $n$, siendo $n=2$ para el sistema estudiado en este trabajo. El rango de la Ecuación (8) sólo depende de $\gamma$, que se expresa como:

$$
\gamma(\hat{\mathbf{x}})=-\frac{I K_{1}^{\prime} K_{5} \hat{x}_{1}}{3 s^{o p t}\left(1-\frac{s^{o p t}-\hat{x}_{2}}{s^{o p t}}\right)} .
$$

La condición que garantiza que $\operatorname{rank}(\mathcal{O})=2$ es

$$
\gamma(\hat{\mathbf{x}}) \neq 0
$$

Como $K_{1}^{\prime}$ y $K_{5}$ son parámetros constantes y distintos que cero (consultar el Apéndice A), la condición (10) se cumple si $x_{1}, x_{2}$ y $I_{\text {stack }}$ son distintos que cero.

Una vez garantizada la observabilidad, las funciones $g_{1} \quad$ y $g_{2}$ en la Ecuación (4) se obtienen del siguiente modo [2]:

$$
\mathbf{g}(\hat{\mathbf{x}})=\left[\begin{array}{l}
g_{1}(\hat{\mathbf{x}}) \\
g_{2}(\hat{\mathbf{x}})
\end{array}\right]=\mathcal{O}(\hat{\mathbf{x}})^{-1}\left[\begin{array}{ll}
0 & 1
\end{array}\right]^{T}
$$

lo cual resulta en

$$
\begin{aligned}
& g_{1}(\hat{\mathbf{x}})=0 \\
& g_{2}(\hat{\mathbf{x}})=\frac{3 I \hat{x}_{2}}{K_{1}^{\prime} K_{5} \hat{x}_{1}} .
\end{aligned}
$$




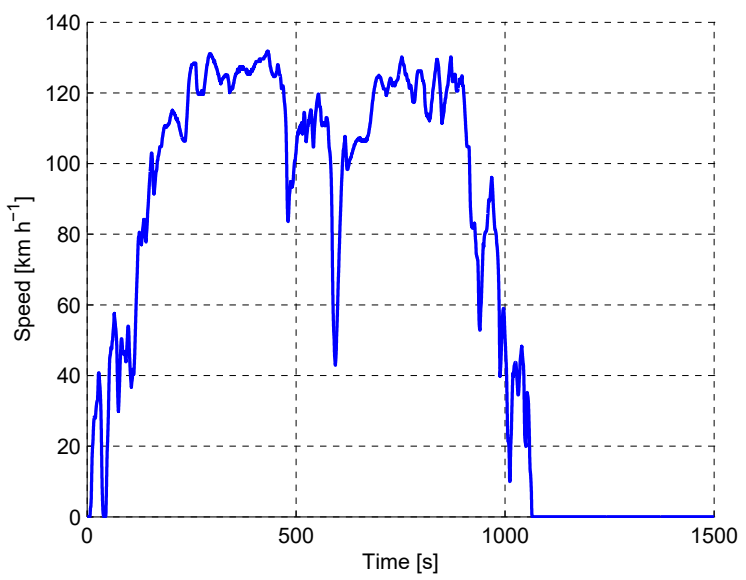

Figura 3: Ciclo de conducción ARTEMIS

Esta selección garantiza que el grado relativo de la salida medida, $T_{f c}$, y la acción de control del observador, $u_{2}$, presentan un grado relativo igual al orden de la planta. Ello implica que no existirá dinámica cero y se podrá influir sobre todas las variables de estado del sistema.

Por lo tanto, considerando (12)-(13), la Ecuación (4) puede ser expresada del siguiente modo:

$$
\begin{aligned}
& \dot{\hat{x}}_{1}=K_{1} I-K_{1}^{\prime} I V_{\text {stack }}+\nu \\
& \dot{\hat{x}}_{2}=K_{3} I-K_{4} f_{p}\left(\hat{x}_{1}\right) \hat{x}_{2}-f_{d}\left(\hat{x}_{2}\right)+\frac{3 I \hat{x}_{2}}{K_{1}^{\prime} K_{5} \hat{x}_{1}} u_{2} \\
& \hat{y}=\hat{x}_{1} .
\end{aligned}
$$

\subsection{Diseño de la acción de corrección}

Como el único estado medido es $T_{f c}$, el error de observación $e_{y}$ se expresa como la diferencia entre la salida estimada $\hat{y} \mathrm{y}$ el vector de salida medido $y$ :

$$
e_{y}=\hat{y}-y=\hat{x}_{1}-x_{1} .
$$

Para llevar la Ecuación (15) a cero en tiempo finito, se implementa una entrada de corrección $u_{2}$, basada en modos deslizantes casi-continuos de segundo orden [6]:

$$
u_{2}=-\beta \frac{\dot{e}_{y}+\left|e_{y}\right|^{2} \operatorname{sign}\left(e_{y}\right)}{\left|\dot{e}_{y}\right|+\left|e_{y}\right|^{1 / 2}},
$$

donde $\beta$ es la ganancia del observador $\left(\beta=0,5 e^{-3}\right.$ para los resultados mostrados en la Sección 5).

La implementación de esta ley de corrección requiere la estimación de la derivada de $e_{y}$. En la actualidad se han propuestos varios algoritmos de derivación robustos que pueden ser fácilmente utilizados con este propósito.

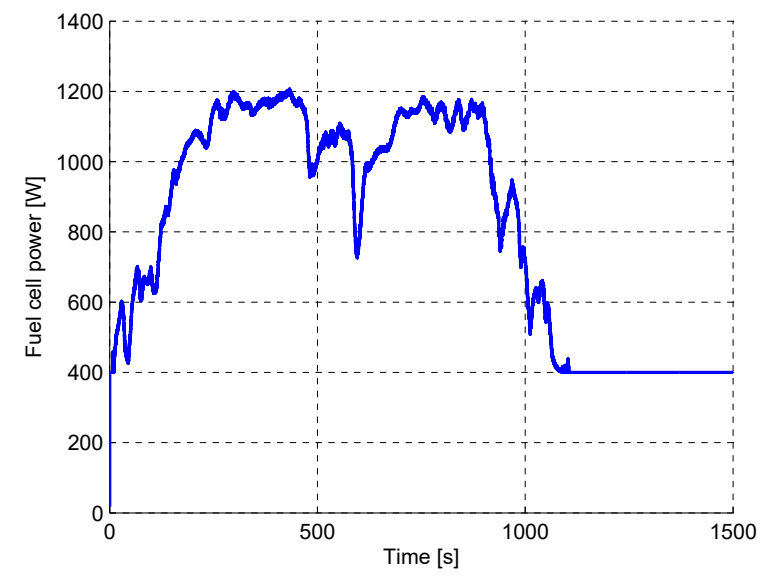

Figura 4: Perfil de potencia necesario para el seguimiento del perfil ARTEMIS (Figura 3)

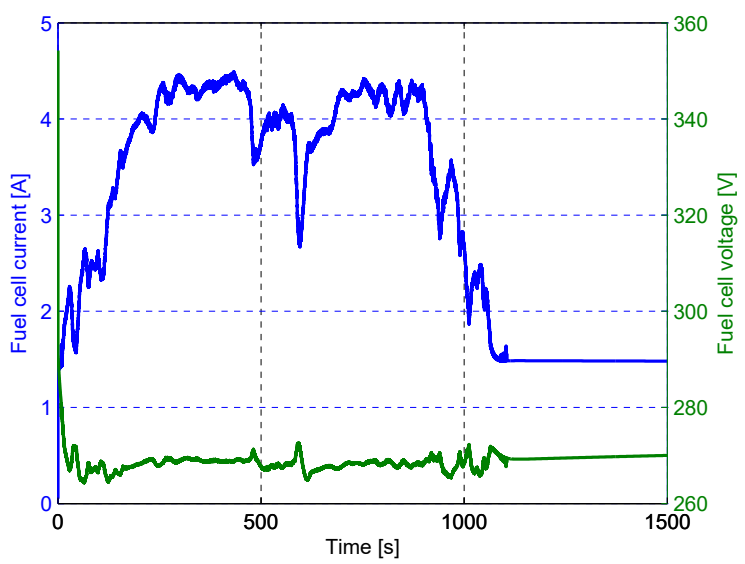

Figura 5: Perfil de tensión y corriente de la pila de combustible seguimiento del perfil ARTEMIS (Figura 3)

\section{RESULTADOS}

En esta sección se mostrarán los resultados obtenidos al utilizar la pila descrita en la sección 2 pero adaptada a una aplicación de tracción [9]. En la simulación se ha substituido las 24 celdas de la H-100 disponible en el laboratorio por 370 celdas. Este es el mismo número de celdas que utiliza la pila de combustible utilizada en el Toyota Mirai.

En esta sección se analizará el comportamiento del observador cuando el vehículo sigue un perfil de velocidad ARTEMIS (Figura 3). La Figura 4 muestra el perfil de potencia necesario para que el vehículo siga el perfil indicado. Se asume la existencia de un controlador de bajo nivel de la pila, no descrito en este trabajo, que fuerza que la pila sea capaz de aportar la potencia deseada para el seguimiento del perfil de velocidad. La Figura 5 muestra la corriente y la tensión de la pila durante el seguimiento del perfil. La corriente corresponde 
a una entrada del modelo mientras que la salida corresponde a una de las salidas.

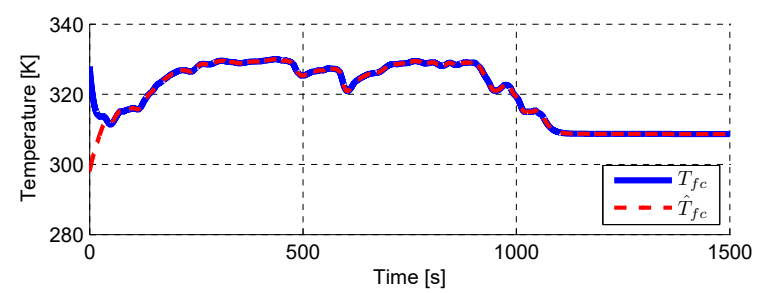

Figura 6: Evolución de la temperatura de la pila de combustible y la estimación del observador durante el seguimiento del perfil ARTEMIS (Figura 3)

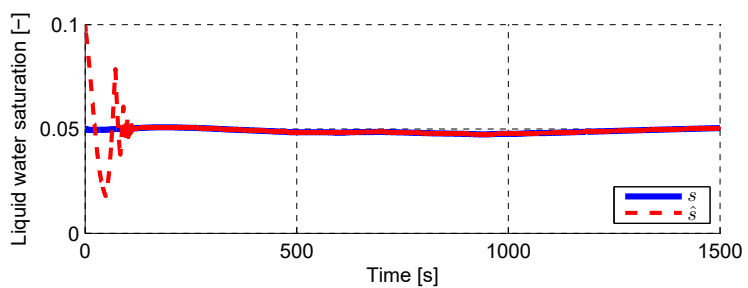

Figura 7: Evolución de la saturación de agua de la pila de combustible y la estimación del observador durante el seguimiento del perfil ARTEMIS (Figura 3)

La Figura 6 muestra la evolución de la temperatura de la pila, $T_{f c}$ y su estimación $\hat{T}_{f c}$. Similarmente, la Figura 7 muestra la evolución de la fracción de agua líquida, $s$, y de su estimación, $\hat{s}$. Como se puede observar, después de un pequeño transitorio la estimación converge a la variable de estado, y una vez se ha producido la convergencia ambas variables permanecen juntas. La Figura 8 muestra la acción de control, $u_{2}$, utilizada para forzar la convergencia de la estimación del estado, $\hat{\mathbf{x}}$, al estado $\mathbf{x}$ del modelo. Como se puede observar, después de un pequeño transitorio la variable conmuta entre dos valores continuamente. Esta conmutación es la que garantiza que las variables de estado del observador se mantengan sobre la superficie de deslizamiento que garantiza que el estado estimado sea igual al estado del modelo.

\section{CONCLUSIONES}

En este trabajo se ha presentado el desarrollo de un observador no lineal para la estimación de la cantidad de agua acumulada en el interior de una pila de combustible comercial. La estimación de este parámetro, no medible, es de vital importancia para caracterizar el estado de la pila.

En la actualidad se están realizando la codificación de los algoritmos en el sistema real con el objetivo

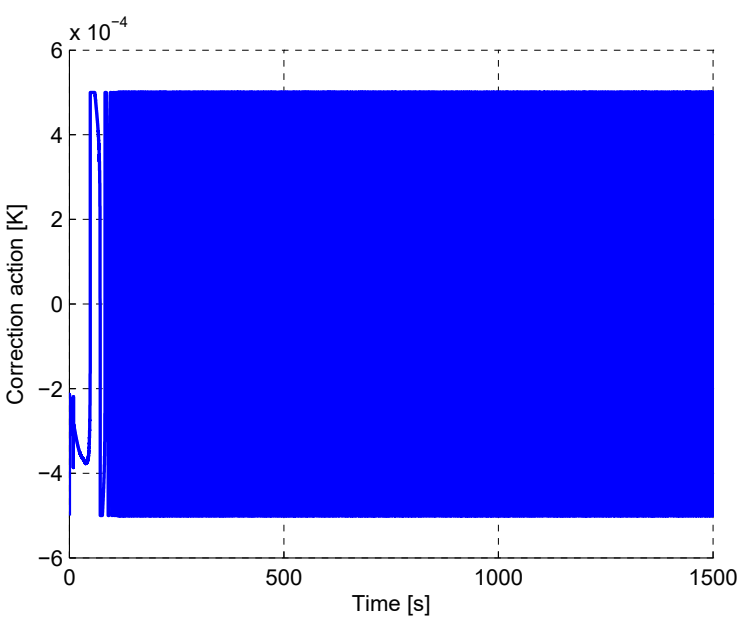

Figura 8: Evolución de la acción de control del observador durante el seguimiento del perfil ARTEMIS (Figura 3).

de validar experimentalmente los desarrollos realizados.

\section{Apéndice A Detalles del modelo}

\section{A.1 Parámetros del modelo}

$$
\begin{aligned}
K_{1} & =\frac{E_{t h} n_{c e l l}}{m_{f c} C_{p, f c}} \\
K^{\prime}{ }_{1} & =\frac{1}{m_{f c} C_{p, f c}} \\
K_{2} & =\frac{\rho_{\text {air }} A_{\text {inlet }} C_{p, a i r}}{m_{f c} C_{p, f c}} \\
K_{3} & =\frac{M_{H_{2} O}}{2 F A_{\text {geo }} \epsilon_{\text {eff }} d_{C L} \rho_{l} K_{\text {sorp }}} \\
K_{4} & =\frac{K_{\text {evap }} M_{H_{2} O}}{R A_{\text {pore }} \epsilon_{\text {eff }} d_{C L} \rho_{l} K_{\text {sorp }}} \\
K_{5} & =\frac{n_{\text {cell }} R}{\alpha n F} \\
K_{6} & =n_{\text {cell }} R_{\text {ohm }} \\
K_{7} & =n_{\text {cell }} E_{0}
\end{aligned}
$$

$$
\begin{aligned}
f_{p}\left(T_{f c}\right) & =\frac{1}{T_{f c}}\left[p^{0} \exp \left(\frac{-E_{a}}{k_{B} T_{f c}}\right)-p^{v}\right] \\
f_{d}(s) & =-\frac{J_{d i f f}}{\epsilon_{e f f} d_{C L} \rho_{l} K_{\text {sorp }}}
\end{aligned}
$$

\section{Agradecimientos}

Este trabajo ha sido parcialmente financiado por los proyectos MINECO/FEDER DPI2015-69286C3-2-R y INN-BALANCE H2020-JTI-FCH-2016$1-735969$. 
Tabla 1: Valores de las principales constantes físicas

\begin{tabular}{|c|c|c|}
\hline Parámetro & Valor & Unidades \\
\hline Capacidad térmica específica de la pila de combustible, $C_{n}$ fc & 1200 & $\mathrm{~J} \cdot \mathrm{kg}^{-1} \mathrm{~K}^{-1}$ \\
\hline Masa de la pila de combustible, $m_{f c}$ & 0,3 & $\mathrm{~kg}$ \\
\hline Densidad del aire @ $20^{\circ} \mathrm{C}, \rho_{\text {air }}$ & 1,205 & $\mathrm{~kg} \cdot \mathrm{m}^{-3}$ \\
\hline Capacidad calorífica específica del aire @ $20^{\circ} \mathrm{C}, C_{p, \text { air }}$ & 1005 & $\mathrm{~J} \cdot \mathrm{kg}^{-1} \cdot \mathrm{K}^{-1}$ \\
\hline Sección eficaz de la estructura del cátodo, $A_{\text {inlet }}$ & $8,5 e^{-3}$ & $\mathrm{~m}^{2}$ \\
\hline Potencial teórico @ $T^{r e f}=25^{\circ} \mathrm{C}$ y $P^{r e f}=1 \mathrm{~atm}, E_{t h}$ & 1,23 & $\mathrm{~V}$ \\
\hline Número de celdas en la pila, $n_{\text {cell }}$ & 370 & - \\
\hline Densidad de corriente de intercambio intrínseco del $\mathrm{Pt}, i_{0}^{\text {ref }}$ & $5 e^{-3}$ & $\mathrm{~A} \cdot \mathrm{m}^{-2}$ \\
\hline Coeficiente de transferencia de carga, $\alpha$ & 0,28 & - \\
\hline Barrera de activación para el ORR en $\mathrm{Pt}, \Delta G^{*}$ & 70000 & $\mathrm{~J} \cdot \mathrm{mol}^{-1}$ \\
\hline Saturación óptima alcanzable del agua líquida, $s^{\text {opt }}$ & 0,165 & - \\
\hline Superficie geométrica del catalizador, $A_{g e o}$ & $22,5 e^{-4}$ & $\mathrm{~m}^{2}$ \\
\hline Resistencia óhmica de la pila, $R_{o h m}$ & 0,7 & $\Omega$ \\
\hline Presión parcial de oxígeno en el cátodo, $p_{\mathrm{O}_{2}}$ & $0,21 \cdot P^{r e f}$ & $\mathrm{~Pa}$ \\
\hline Porosidad efectiva, $\epsilon_{e f f}$ & 0,5 & - \\
\hline Permeabilidad efectiva, $K_{e f f}$ & $1 e^{-14}$ & $\mathrm{~m}^{2}$ \\
\hline Densidad de ag & 970 & $\mathrm{~kg} \cdot \mathrm{m}^{-3}$ \\
\hline Viscosidad del a & $3,517 e^{-4}$ & $\mathrm{~Pa} \cdot \mathrm{s}$ \\
\hline Tensión superficial líquida del agua, $\sigma$ & 0,0625 & $\mathrm{~N} \cdot \mathrm{m}^{-1}$ \\
\hline Ángulo efectivo de contacto, $\theta$ & 91 & o \\
\hline Espesor eficaz de los medios de difusión, $d_{\text {diff }}$ & $0,41 e^{-3}$ & $\mathrm{~m}$ \\
\hline Volumen de la capa catalítica, $V_{C L}$ & $2,25 e^{-8}$ & $\mathrm{~m}^{3}$ \\
\hline Espesor de la capa catalítica, $d_{C L}$ & $0,01 e^{-3}$ & $\mathrm{~m}$ \\
\hline Constante de tiempo de absorción, $K_{\text {sorp }}$ & 360 & - \\
\hline Constante de tiempo de evaporación, $K_{\text {evap }}$ & $8,6 e^{5}$ & - \\
\hline Factor pre-exponencial, $p^{0}$ & $1,196 e^{11}$ & $\mathrm{~Pa}$ \\
\hline Energía de activación de la evaporación, $E_{a}$ & 0,449 & $\mathrm{eV}$ \\
\hline Constante de Boltzmann, $k_{B}$ & $8,617 e^{-5}$ & $\mathrm{eV} \cdot \mathrm{K}^{-1}$ \\
\hline Presión ambiente del cátodo, $P_{a m b}$ & $1,013 e^{5}$ & $\mathrm{~Pa}$ \\
\hline Temperatura ambiente del cátodo, $T_{a m b}$ & 298 & $\mathrm{~K}$ \\
\hline Presión de vapor ambiente del cátodo @ $75 \% R H, p^{v}$ & 2380 & $\mathrm{~Pa}$ \\
\hline Superficie de poro por unidad de volumen, $A_{\text {pore }}$ & $2 e^{7}$ & $\mathrm{~m}^{2} \cdot \mathrm{m}^{-3}$ \\
\hline
\end{tabular}




\section{Referencias}

[1] Bavarian, M., Soroush, M., Kevrekidis, I. G., And Benziger, J. B. Mathematical modeling, steady-state and dynamic behavior, and control of fuel cells: a review. Industrial $\&$ engineering chemistry research 49 , 17 (2010), 7922-7950.

[2] Davila, J., Fridman, L., Pisano, A., And UsAi, E. Finite-time state observation for non-linear uncertain systems via higher-order sliding modes. International Journal of Control 82, 8 (2009), 1564-1574.

[3] FuelCell store. H-100 Fuel Cell Stack. User Manual.

[4] He, W., Lin, G., and Van Nguyen, T. Diagnostic tool to detect electrode flooding in proton-exchange-membrane fuel cells. AIChE Journal 49, 12 (2003), 3221-3228.

[5] Jensen, S. H., Larsen, P. H., and MoGENSEN, M. Hydrogen and synthetic fuel production from renewable energy sources. International Journal of Hydrogen Energy 32, 15 (2007), 3253-3257.

[6] Levant, A. Quasi-continuous high-order sliding-mode controllers. In Decision and Control, 2003. Proceedings. 42nd IEEE Conference on (2003), vol. 5, IEEE, pp. 46054610 .

[7] Luna, J., Jemei, S., Yousfi-Steiner, N., Husar, A., Serra, M., and Hissel, D. Nonlinear predictive control for durability enhancement and efficiency improvement in a fuel cell power system. Journal of Power Sources 328 (2016), 250-261.

[8] Luna, J., Usai, E., Husar, A., and SeRRA, M. Nonlinear observation in fuel cell systems: A comparison between disturbance estimation and high-order sliding-mode techniques. International Journal of Hydrogen Energy 41, 43 (2016), 19737-19748.

[9] Mayur, M., Strahl, S., Husar, A., and Bessler, W. G. A multi-timescale modeling methodology for pemfc performance and durability in a virtual fuel cell car. International Journal of Hydrogen Energy 40, 46 (2015), 16466 - 16476.

[10] Pasaogullari, U., and Wang, C. Liquid water transport in gas diffusion layer of polymer electrolyte fuel cells. Journal of the Electrochemical Society 151, 3 (2004), A399A406.

[11] Petrone, R., Zheng, Z., Hissel, D., Péra, M.-C., Pianese, C., Sorrentino, M., Becherif, M., And Yousfi-Steiner, $\mathrm{N}$. A review on model-based diagnosis methodologies for pemfcs. International Journal of Hydrogen Energy 38, 17 (2013), 7077-7091.

[12] Sharaf, O. Z., and Orhan, M. F. An overview of fuel cell technology: Fundamentals and applications. Renewable and Sustainable Energy Reviews 32 (2014), 810-853.

[13] Slotine, J.-J. E., Li, W., Et Al. Applied nonlinear control, vol. 199. prentice-Hall Englewood Cliffs, NJ, 1991.

[14] Strahl, S., and Costa-Castelló, R. Model-based analysis for the thermal management of open-cathode proton exchange membrane fuel cell systems concerning efficiency and stability. Journal of Process Control 47 (2016), 201-212.

[15] Strahl, S., and Costa-Castelló, R. Model-based analysis for the thermal management of open-cathode proton exchange membrane fuel cell systems concerning efficiency and stability. Journal of Process Control 47 (2016), $201-212$.

[16] Strahl, S., Husar, A., Puleston, P., AND RierA, J. Performance improvement by temperature control of an open-cathode pem fuel cell system. Fuel Cells (2014), 1-3.

[17] Strahl, S., Husar, A., Riera, J., And Costa Castelló, R. Control de temperatura en pilas de combustible tipo pem de cátodo abierto. In Actas de las XXXV Jornadas de Automática (Valencia, Septiembre 2014), Comité Español de Automática (CEA-IFAC), pp. 215-221. ISBN-13: 978-84-697-0589-6.

[18] Yousfi-Steiner, N., Moçotéguy, P., Candusso, D., Hissel, D., Hernandez, A., And Aslanides, A. A review on pem voltage degradation associated with water management: Impacts, influent factors and characterization. Journal of power sources 183, 1 (2008), 260-274. 